\title{
Middle Longitudinal Fasciculus in the Human Brain from Fiber Dissection
}

\author{
Igor Lima Maldonado ${ }^{1,2}$, Ilyess Zemmoura ${ }^{3,4}$, \\ Christophe Destrieux ${ }^{3-4}$
}

\begin{abstract}
Although the Middle Longitudinal Fasciculus (MdLF) is not part of the insular lobe, it penetrates the temporal operculum, which is manipulated or partially removed during surgical approaches to the insula. We present a comprehensive description of that fascicle and its anatomical relationships with neighboring structures that have been described in previous chapters. It has been hypothesized that MdLF plays a role in attention and language processing, however studies with peroperative subcortical electrostimulation or temporal lobectomies did not demonstrate permanent language deficits following resection of its anterior segment. The MdLF has close relationships to adjacent bundles, such as the arcuate fasciculus and the inferior frontooccipital fasciculus that are required to be recognized and differentiated for adequate interpretation of tractography images and surgical planning.
\end{abstract}

Keywords: fiber bundles, anatomy, middle longitudinal fasciculus, temporal operculum.

\footnotetext{
1. Universidade Federal da Bahia, , Instituto de Ciências da Saúde, Departamento de Biomorfologia, Salvador-Bahia, Brazil

2. Universidade Federal da Bahia, Complexo Hospitalar Universitário Prof. Edgard Santos, Serviço de Neurocirurgia, Salvador-Bahia, Brazil

3. Université François-Rabelais de Tours, Inserm, Imagerie et cerveau UMR 930, Tours, France

4. CHRU de Tours, Tours, France
} 


\section{Introduction}

Although the Middle Longitudinal Fasciculus (MdLF) is not part of the insular lobe, it penetrates the temporal operculum, which is manipulated, or partially removed in surgical approaches to the insula. In this chapter, we present a comprehensive description of that fascicle and its anatomical relationships with neighboring structures that were previously described in Chapters 2 and 3. For the purpose of demonstrating the anatomy, a variant of the Klingler's technique for fiber dissection was used.

This bundle was initially described in the rhesus monkey. First identified by radioisotopic tracing in the 1980s [1,2], the MdLF was subsequently studied using diffusion imaging techniques in a study published in 2007 [3]. In 2008, its trajectory was first traced in humans, using a segmentation technique in MR and tractography [4], and then using fiber dissection techniques [5]. Regarding its trajectory with respect to sulci and gyri, the MdLF extends from the parieto-occipital cortex to anterior portions of the temporal lobe, penetrating the superior temporal gyrus.

\section{Topography and trajectory}

A stepwise dissection of the temporo-parieto-occipital white matter is presented in Figures 4.1 through 4-3. In those specimens, the arachnoid membrane and the vascular elements were already removed. Before dissection, the first step is the study of the individual morphology of the sulci and gyri on the cerebral surface (Figures 2.1A and 3.1A).

Although a relatively constant organization of sulci exists, some variants are often noted. The lateral surface of the temporal lobe is generally divided into three gyri, which are parallel to two identifiable sulci (the superior and inferior temporal sulci). A vertical and relatively deep sulcus is often responsible for an apparent discontinuity, particularly of T1 and/or T3. Addi- 
tionally, cortical folds link T2 to T3 very frequently, and less frequently $\mathrm{T} 1$ to $\mathrm{T} 2$.

In a majority of specimens, the anterior portion of the inferior parietal lobule (IPL) is limited anteriorly by the postcentral sulcus. The supramarginal gyrus (GSM) begins with a narrow cortical fold that joins to the inferior extremity of the postcentral gyrus. With an irregular trajectory, it contours the posterior extremity (posterior ramus) of the lateral sulcus. A second and frequently interrupted gyrus, the angular gyrus (AG), curves around the posterior extremity of the superior temporal sulcus. The intermedius primus (Jensen's) sulcus may be interposed between these two gyri (SMG and AG) with variable depth and length. A pre-AG variant may also potentially be observed in some cases. In this variant, a narrow gyrus is interposed between the AG and the SMG [6]. A pre-SMG variant, comprising a narrow gyrus intercalated between the postcentral gyrus and the SMG, is less frequently observed.

As expected, the Klingler's technique renders the cortical gray matter brittle and friable, and it may be removed gently and progressively. At the subcortical level, the short U-shaped association fibers may be easily exposed on the lateral side of the temporal lobe and the IPL. This gradual removal allows exposure of the $\mathrm{AF}$ in the postero-superior portion of the temporal lobe and in the depth of the SMG. Note that at this stage, T1 and white matter of AG were still kept in place and partially preserved (Figure 4.1A).

Once the dissection of the lateral aspect has been completed, the AF may be sectioned in its postero-superior portion, medial to the parietal operculum, raised inferiorly and partially removed (Figure 4.1B). The sagittal stratum is then exposed in contact to the medial aspect of the arcuate fasciculus.

At the level of the most superficial layer of the SS, the Middle Longitudinal Fasciculus is viewed as a group of horizontal fibers joining the white matter of the superior temporal gyrus (Figures 4.1B and 4.2). They may be followed forward to the caudal portion of the anterior third of T1. Frequently, additional 
small groups potentially join the same gyrus at adjacent points. Posteriorly; its fibers are partially covered by the thick vertical portion of the SLF/FA complex. Subsequent dissection highlights groups of such fibers continuing beyond the posterior limits of the IPL (Figure 4.2). There is significant discussion about the posterior terminations of the MdLF and some variation seem to exist, however the angular gyrus, superior parietal lobule and occipital lobe (lateral and medial aspects) are most commonly reported $[7,8,5,9,10]$.

\section{Relationships to adjacent fiber bundles}

The MdLF presents a global organization in three segments. The posterior segment is the longest and widest on the sagittal plane. At this level, the fibers are distributed in a thin layer, located medial with respect to SLF/AF. In the middle segment, the bundle leaves the SS to penetrate the posterior and deep portion of T1. Here, its fibers are relatively compacted, and the bundle has a narrower appearance. Finally, the anterior segment is the one in which the MdLF integrates the white matter of the temporal operculum.

The inferior fronto-occipital fasciculus (IFOF) may be identified in the same specimen. In this step, the operator confirms whether the previously dissected fibers are independent from neighboring fasciculi. The deep temporal white matter (deeper to the dissected T1) is explored to demonstrate the IFOF. Additional dissection may expose the uncinate fasciculus and centrifugal fibers of the postero-superior portion of the external capsula. With this procedure, the IFOF is located deeper than the fibers that penetrate T1. While the IFOF tilts inward to the insular lobe and deeply reach the external capsule, the MdLF does not. Its fibers remain at a relatively superficial level and adopt a much less curved trajectory. Inside the SS, the IFOF is partially covered by the MdLF (Figure 4-4). 


\section{Surgical and radiological considerations}

The first observations of an MdLF were obtained in radioisotopic tracing studies in the rhesus monkey $[4,11]$. It has only more recently been observed that DTI techniques bring arguments of its existence in humans [4]. Tractography has also been used to distinguish adjacent bundles from the MdLF. Since this fasciculus could provide connections to the posterior part of $\mathrm{T} 1$, the hypothesis of a role in language and attention has been formulated.

Considering the trajectory of the MdLF, it has been hypothesized that it has a role in attention processing. Some authors suggested that it could be an important pathway for linguistic information in the dominant hemisphere [4]. Despite this, in patients in which peroperative subcortical electrostimulation was used in the topography of the anterior segment of the MdLF of the dominant hemisphere; no linguistic interference was induced [12]. In addition, no permanent language deficit occurred despite resection of the anterior segment as routinely performed during temporal lobectomy. It may be inferred then that the MdLF potentially plays a role that does not seem to be essential for language processing. As a consequence, at least its anterior segment can be removed safely. In addition, adequate recognition of the elements of the white matter anatomy is important in order to avoid confusion with adjacent bundles, such as the IFOF, while interpreting tractography images or planning surgical approaches to the temporal operculum and insula.

\section{Conclusion}

The study of the descriptive and topographic anatomy of the middle longitudinal fasciculus allows an overview of concepts that are important for neuroimaging and neurosurgery. A strong understanding of the white matter anatomy is the basis 
for precise interpretation of MRI tractography examinations and for surgical planning of parenchymal approaches. 


\section{References}

1. Schmahmann JD, Pandya DN (2006) Fiber pathways of the brain. Oxford University Press, Inc., Oxford

2. Seltzer B, Pandya DN (1984) Further Observations on ParietoTemporal Connections in the Rhesus Monkey. Experimental Brain Research 55:301-312

3. Schmahmann JD, Pandya DN, Wang R, Dai G, D'Arceuil HE, De Crespigny AJ, Wedeen VJ (2007) Association fibre pathways of the brain: parallel observations from diffusion spectrum imaging and autoradiography Brain 130:630-653

4. Makris N, Papadimitriou GM, Kaiser JR, Sorg S, Kennedy DN, Pandya DN (2008) Delineation of the Middle Longitudinal Fascicle in Humans: A Quantitative, In Vivo, DT-MRI Study. Cerebral Cortex. doi:bhn124 [pii]

10.1093/cercor/bhn124

5. Maldonado IL, de Champfleur NM, Velut S, Destrieux C, Zemmoura I, Duffau H (2013) Evidence of a middle longitudinal fasciculus in the human brain from fiber dissection. J Anat 223 (1):38-45. doi:10.1111/joa.12055

6. Kiriyama I, Miki H, Kikuchi K, Ohue S, Matsuda S, Mochizuki T (2009) Topographic Analysis of the Inferior Parietal Lobule in High-Resolution 3D MR Imaging. AJNR Am J Neuroradiol 30 (3):520-524. doi:10.3174/ajnr.A1417

7. Makris N, Papadimitriou GM, Kaiser JR, Sorg S, Kennedy DN, Pandya DN (2009) Delineation of the Middle Longitudinal Fascicle in Humans: A Quantitative, In Vivo, DT-MRI Study. Cerebral Cortex 19:777--785. doi:bhn124 [pii]

10.1093/cercor/bhn124 
8. Menjot N, Maldonado IL, Moritz-Gasser S, Le Bars E, Bonafe A, Duffau H (2013) Middle Longitudinal Fasciculus delineation within language pathways. European Journal of Radiology 82:151-157

9. Makris N, Preti MG, Wassermann D, Rathi Y, Papadimitriou GM, Yergatian C, Dickerson BC, Shenton ME, Kubicki M (2014) Human middle longitudinal fascicle: segregation and behavioralclinical implications of two distinct fiber connections linking temporal pole and superior temporal gyrus with the angular gyrus or superior parietal lobule using multi-tensor tractography. Brain Imaging Behav 7 (3):335-352.

doi:10.1007/s11682-013-9235-2

10. Makris N, Preti MG, Asami T, Pelavin P, Campbell B, Papadimitriou GM, Kaiser J, Baselli G, Westin CF, Shenton ME, Kubicki M (2014) Human middle longitudinal fascicle: variations in patterns of anatomical connections. Brain Struct Funct 218 (4):951-968. doi:10.1007/s00429-012-0441-2

11. Ribas GC, Oliveira E (2007) [The insula and the central core concept]. Arq Neuropsiquiatr 65 (1):92-100. doi:S0004282X2007000100020 [pii]

12. De Witt Hamer P, Moritz-Gasser S, Gatignol P, Duffau H (2010) Is the human left middle longitudinal fascicle essential for language? A brain electrostimulation study. Human Brain Mapping 32 (6):962-973

\section{FIGURE LEGENDS}

Figure 4.1. Exposure of the Middle Longitudinal Fasciculus using fiber dissection. The cortex and short association fibers were removed and long association pathways were exposed $(\boldsymbol{A})$. Part of the Superior Longitudinal Fasciculus - Arcuate Fasciculus complex is seen. To explore the association bundles medially to 
it, the arcuate fasciculus was sectioned (arrow) and removed (B).

Figure 4.2. Deep dissection. The MdLF fibers travel in the superficial layer of the sagittal stratum and penetrate the superior temporal gyrus. Reproduced (with permission) from Maldonado et al. Evidence of a middle longitudinal fasciculus in the human brain from fiber dissection. J Anat 2013; 223(1): 38-45. [5]

Figure 4.3. The inferior Fronto-Occipital Fasciculus (iFOF) was exposed and the Middle Longitudinal Fasciculus (MdLF) was reflected with part of the superior temporal gyrus. The fibers of iFOF penetrates deeper than those of MdLF and reach the antero-inferior portion of the external capsule, posterior to the uncinate fasciculus and the limen of the insula. 